\title{
Regionalização da lâmina suplementar de irrigação e época de plantio da cultura de feijão, no Estado de Goiás ${ }^{1}$
}

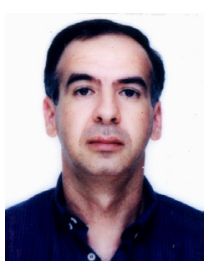

Luiz F. C. de Oliveira² \& Daniel F. de Carvalho ${ }^{3}$

\author{
1 Trabalho financiado pela FUNAPE-UFG \\ ${ }^{2}$ Escola de Agronomia e Engenharia de Alimentos da UFG. Campus II, CP 131, CEP 74001-970, Goiânia, GO. \\ Fone: (62) 521-1534, Fax: (62) 521-1552. E-mail: Ifco@agro.ufg.br (Foto) \\ 3 Instituto de Tecnologia/Departamento de Engenharia da UFRRJ. BR 465, km 7, CEP 23851-970, Seropédica, RJ. \\ Telefax: (21) 2682-1860. Email: carvalho@ufrrj.br
}

Protocolo 87 - 27/6/2002 - Aprovado em 14/4/2003

\begin{abstract}
Resumo: Objetivou-se, através deste trabalho, a estimativa e a regionalização da lâmina suplementar de irrigação e definição da melhor época de plantio para a cultura de feijão de verão, safrinha e de inverno, no Estado de Goiás; para tal, empregou-se o software DEMANDA e uma série histórica dos elementos do clima de estações meteorológicas cadastradas pelo Instituto Nacional de Meteorologia, para o Estado de Goiás e estados limítrofes. A adoção da irrigação suplementar e a definição da melhor época de plantio para a cultura de feijão, permitiram uma redução média na demanda total da quantidade de água a ser aplicada na irrigação, nos plantios de verão, safrinha e inverno, de 446,$1 ; 235,7$ e $66,0 \%$, respectivamente. Os resultados obtido neste trabalho permitiu a geração de mapas temáticos que podem servir de orientação na elaboração de sistemas de irrigação de menor porte, redução no consumo de energia e na quantidade de água necessária à irrigação.
\end{abstract}

Palavras-chave: uso consuntivo, evapotranspiração, precipitação

\section{Regionalization of the supplemental irrigation depth and planting time of bean crop in the State of Goiás, Brazil}

\begin{abstract}
This work had as objectives of estimation and the regionalization of the supplemental depth of irrigation as well as definition of the best planting time for the summer, fall and winter bean crop in the State of Goiás. For the purpose software DEMANDA and historical series registered by the National Institute of Meteorology for the State of Goiás and bordering states were used. The adoption of the supplemental irrigation and the definition of the best planting time for the summer, fall seeded and winter bean crop allowed a mean reduction in the total demand of water to be applied of $446.1 ; 235.7$ and $66.0 \%$, respectively. The results obtained in this work allowed to obtain thematic maps which may serve as orientation in the elaboration of overhead irrigations of smaller load, reduction in the consumption of energy and in the amount of water necessary for the irrigation.
\end{abstract}

Key words: consumptive use, evapotranspiration, precipitation

\section{INTRODUÇ̃̃O}

A quantidade de água necessária à irrigação resulta de um balanço que se faz para um período entre a evapotranspiração da cultura e as precipitações. Quando realizadas, as irrigações devem ser programadas de forma a suplementar as deficiências de água decorrentes da má distribuição temporal e da forma aleatória com que ocorrem as precipitações.

Os principais fatores que influenciam na quantidade de água requerida pelas plantas são os climáticos, as características das plantas, as práticas culturais e o tipo de solo, sendo também as principais técnicas para se estimar o requerimento de água pelas plantas baseadas em dados climáticos (Sediyama, 1996). É significativa, portanto, a obtenção de dados climáticos regionais confiáveis visando às estimativas mais precisas da evapotranspiração e ao melhor aproveitamento das precipitações naturais no dimensionamento de sistemas de irrigação.

Segundo Sousa (1993) registros históricos de dados climáticos permitem a estimativa da precipitação e da evapotranspiração e, conseqüentemente, o balanço de água no solo pode ser utilizado para se ter, previamente, as demandas total e diária de irrigação suplementar, em função da época de plantio da 
cultura. Com esses dados disponíveis, tem-se a possibilidade de planejar o plantio da cultura, de maneira a se utilizar a precipitação natural com maior eficiência.

No Brasil e de modo geral, os projetos de irrigação são dimensionados em termos de irrigação total, de modo a atender às necessidades nos períodos críticos de água para os cultivos, não se levando em consideração as precipitações naturais o que, logicamente, levaria a um superdimensionamento. A análise da distribuição de chuvas tem sido apresentada em bases probabilísticas para identificar áreas e épocas de plantio com maiores chances de sucesso para a agricultura de sequeiro, bem como para identificar a conveniência do uso da irrigação suplementar. Salienta-se que, quando se adota a prática da irrigação suplementar de forma bem planejada, resultarão em menores investimentos, menores custos operacionais e, em conseqüência, a possibilidade de maiores lucros (Silva, 1982).

Segundo Wolf (1977) citado por Assad et al. (1998) o clima do Planalto Central é caracterizado por uma estação seca bem definida, que se estende de maio a setembro. Esta característica pode ser generalizada para toda a região dos cerrados brasileiros, onde a atividade agrícola se concentra no período chuvoso, quando ocorrem de 80 a $90 \%$ do total anual de chuvas, em torno de $1500 \mathrm{~mm}$. Embora este total seja considerado suficiente para muitas culturas, a deficiência hídrica é um dos fatores limitantes para a agricultura na região dos cerrados.

As grandes flutuações observadas na precipitação mensal sugerem que as precipitações médias não constituem um bom índice para análise da oferta pluviométrica, principalmente porque estas médias estão associadas a coeficientes de variação que chegam a ultrapassar a $100 \%$ em alguns meses menos chuvosos. Portanto, a precipitação provável é de capital importância para o planejamento e dimensionamento de sistemas de irrigação. Neste caso, a precipitação provável se refere à lâmina mínima com determinada probabilidade de ocorrência.

Para o manejo da irrigação e dependendo do comprimento do período em que os dados de precipitação forem agrupados, com base na probabilidade de ocorrência de chuva nos próximos dias, pode-se decidir se deve-se ou não aplicar a lâmina necessária na próxima irrigação (Bernardo \& Hill, 1979; Bernardo, 1995; Costa, 1991 e Sediyama, 1996); assim, é conveniente se optar por um valor de probabilidade de ocorrência compatível com a atividade agrícola. De modo geral, adota-se uma probabilidade de $75 \%$, implicando num risco de $25 \%$, ou seja, de que a cada quatro anos, em média três anos, se estará atendendo, de forma adequada, às necessidades de água das culturas, quando se faz irrigação suplementar.

Segundo Assis (1993) e Bernardo (1995) os dados climatológicos de um local ou região podem ser analisados pela simples inspeção dos registros históricos ou, alternativamente, pelo ajuste de um modelo teórico a esses dados. O modelo teórico apresenta a vantagem de sintetizar as informações em alguns parâmetros de uma equação, o que resulta na facilidade de seu manuseio. Um modelo teórico para se descrever a variabilidade da chuva pode ser desenvolvido em duas etapas, em que a primeira reproduz a condição de ocorrência e a segunda representa a magnitude da chuva. A distribuição de probabilidade gama, segundo Assis (1993), Assis et al. (1996), Costa
(1991) e Sediyama et al. (1995), pode ser considerada a mais adequada para representar a magnitude da chuva de períodos curtos.

Modelos não espaciais são baseados no conceito de que o valor mensurado pela estação meteorológica representa uma área homogênea ao redor da mesma, o que, genericamente, não é verdade em áreas com grandes variações climáticas e topográficas. Esta aproximação proporciona erros na estimativa dos parâmetros climatológicos em escala regional (Sousa, 1993).

Nos últimos anos, praticamente todos os estudos climáticos da região dos cerrados apresentaram caráter local sendo, assim, de abrangência limitada. Segundo Assad et al. (1993) vários trabalhos de caráter localizado foram realizados nesta região de estudo, sem a preocupação de espacializar o fenômeno, tanto em termos de freqüência como em termos probabilísticos. Normalmente, a análise dos totais anuais não é suficiente para aplicação, uma vez que é fundamental, também, se conhecer a distribuição e a freqüência de ocorrência da precipitação e regionalizá-la em forma de cartogramas, mapas e isolinhas. Desta maneira, a utilização de um sistema de informação geográfica se faz necessário, principalmente se o interesse é regionalizar as informações pluviométricas.

Conforme Nascimento (1998) a cultura do feijoeiro tem-se constituído na principal alternativa de cultivo irrigado em Goiás, contribuindo com $90 \%$ da produção do estado, com uma área plantada na safra 1999-2000 de 120.000 ha, sendo 40.000 irrigados. Em vista do exposto, este trabalho teve como objetivo a estimativa e a regionalização da lâmina suplementar de irrigação e definição da melhor época de plantio para a cultura de feijão de verão, safrinha e de inverno, no Estado de Goiás.

\section{MATERIAL E MÉTODOS}

Empregou-se, nesse estudo, uma série histórica de 15 anos de observações diárias dos elementos do clima de estações meteorológicas cadastradas pelo Instituto Nacional de Meteorologia (INMET) existentes no Estado de Goiás. Para melhorar a espacialização das informações, selecionaram-se, também, estações dos Estados limítrofes (Bahia, Distrito Federal, Mato Grosso, Mato Grosso do Sul e Tocantins) totalizando 27 estações. Na Tabela 1 tem-se as estações selecionadas com os respectivos números de cadastro no INMET, além das coordenadas geográficas.

Utilizando-se o software DEMANDA desenvolvido por Carvalho (1998) e o banco de dados do INMET estimaram-se, para cada estação, os valores diários de evapotranspiração de referência pelo método de Penman-Monteith, e a precipitação provável $(\mathrm{Pp})$ pela distribuição gama com a probabilidade de ocorrência de 75\% (Eqs. 1 e 2).

$$
\begin{aligned}
\text { ETo } & =\frac{\gamma}{\delta+\gamma^{*}}(\mathrm{Rn}-\mathrm{G}) \frac{1}{\lambda} \\
& +\frac{\gamma}{\delta+\gamma^{*}} \frac{900}{\mathrm{~T}+273} \mathrm{u}_{2}\left(\mathrm{e}_{\mathrm{a}}-\mathrm{e}_{\mathrm{d}}\right)
\end{aligned}
$$


Tabela 1. Estações do INMET (Instituto Nacional de Meteorologia) selecionadas

\begin{tabular}{|c|c|c|c|c|}
\hline $\begin{array}{l}\text { Código } \\
\text { INMET }\end{array}$ & Município & Estado & $\begin{array}{l}\text { Latitude } \\
\text { (S) }\end{array}$ & $\begin{array}{l}\text { Longitude } \\
\text { (W) }\end{array}$ \\
\hline 83236 & Barreiras & BA & $12^{0} 09^{\prime}$ & $45^{0} 00^{\prime}$ \\
\hline 83238 & Paratinga & BA & $12^{0} 41^{\prime}$ & $43^{0} 12^{\prime}$ \\
\hline 83076 & Santa Rita de Cássia & $\mathrm{BA}$ & $11^{0} 01^{\prime}$ & $44^{0} 3^{\prime}$ \\
\hline 83579 & Araxá & MG & $19^{0} 34^{\prime}$ & $46^{0} 56^{\prime}$ \\
\hline 83514 & Capinópolis & MG & $18^{0} 41^{\prime}$ & $49^{0} 34^{\prime}$ \\
\hline 83481 & João Pinheiro & MG & $17^{0} 41$ & $46^{0} 10^{\prime}$ \\
\hline 83524 & Monte Alegre de Minas & MG & $18^{0} 52^{\prime}$ & $48^{0} 52^{\prime}$ \\
\hline 83479 & Paracatu & MG & $17^{0} 13$ & $46^{0} 52^{\prime}$ \\
\hline 83531 & Patos de Minas & MG & $18^{0} 34^{\prime}$ & $46^{0} 31^{\prime}$ \\
\hline 83577 & Uberaba & MG & $19^{0} 45$ & $42^{0} 5$ \\
\hline 83615 & Água Clara & MS & $20^{\circ} 27^{\prime}$ & $52^{0} 53^{\prime}$ \\
\hline 83611 & Campo Grande & MS & $20^{\circ} 27^{\prime}$ & $54^{0} 37^{\prime}$ \\
\hline 83512 & Coxim & MS & $18^{0} 03$ & $54^{0} 46^{\prime}$ \\
\hline 83704 & Ivinhema & MS & $22^{0} 19^{\prime}$ & $53^{0} 56^{\prime}$ \\
\hline 83565 & Paranaíba & MS & $19^{0} 42^{\prime}$ & $51^{0} 11^{\prime}$ \\
\hline 83665 & Sangradouro & MT & $15^{0} 38^{\prime}$ & $53^{0} 05^{\prime}$ \\
\hline 83228 & Peixe & TO & $12^{0} 09^{\prime}$ & $45^{0} 00^{\prime}$ \\
\hline 83377 & Brasília & DF & $15^{0} 47^{\prime}$ & $47^{0} 56^{\prime}$ \\
\hline 83368 & Aragarças & GO & $15^{0} 54^{\prime}$ & $52^{0} 14^{\prime}$ \\
\hline 83526 & Catalão & GO & $18^{0} 11$ & $47^{0} 57^{\prime}$ \\
\hline 83379 & Formosa & GO & $15^{0} 32^{\prime}$ & $47^{0} 02^{\prime}$ \\
\hline 83423 & Goiânia & GO & $16^{0} 40^{\prime}$ & $49^{0} 15$ \\
\hline 83374 & Goiás & GO & $15^{0} 55$ & $50^{0} 08^{\prime}$ \\
\hline 83467 & Mineiros & GO & $17^{0} 34^{\prime}$ & $52^{0} 33^{\prime}$ \\
\hline 83332 & Posse & GO & $14^{0} 06^{\prime}$ & $46^{0} 22^{\prime}$ \\
\hline 83376 & Pirenópolis & GO & $15^{0} 51^{\prime}$ & $48^{0} 58^{\prime}$ \\
\hline 83470 & Rio Verde & GO & $17^{0} 48^{\prime}$ & $50^{0} 55^{\prime}$ \\
\hline
\end{tabular}

em que:

ETo - evapotranspiração de referência $\left(\mathrm{mm} \mathrm{d}^{-1}\right)$

$\gamma \quad$ - coeficiente psicrométrico $\left(\mathrm{kPa}^{0} \mathrm{C}^{-1}\right)$

$\delta$ - declividade da curva de pressão de vapor de saturação $\left(\mathrm{kPa}^{0} \mathrm{C}^{-1}\right)$

$\gamma^{*} \quad$ - coeficiente psicrométrico modificado $\left(\mathrm{kPa}^{0} \mathrm{C}^{-1}\right)$

$\mathrm{Rn}$ - saldo de radiação à superfície da cultura $\left(\mathrm{MJ} \mathrm{m}^{-2} \mathrm{~d}^{-1}\right)$

$\mathrm{G} \quad$ - fluxo de calor no solo $\left(\mathrm{MJ} \mathrm{m}^{-2} \mathrm{~d}^{-1}\right)$

$\lambda$ - calor latente de evaporação $\left(\mathrm{MJ} \mathrm{kg}^{-1}\right)$

$\mathrm{T}$ - temperatura média do ar $\left({ }^{\circ} \mathrm{C}\right)$

(ea - ed) - déficit de pressão de vapor $(\mathrm{kPa})$

$\mathrm{u}_{2} \quad$ - velocidade do vento a uma altura de $2 \mathrm{~m}\left(\mathrm{~m} \mathrm{~s}^{-1}\right)$

$$
\begin{gathered}
\mathrm{G}(\mathrm{Y} \leq \text { yo })=\frac{1}{\Gamma(\alpha) \beta^{\alpha}} \int_{0}^{\mathrm{y}_{0}} \mathrm{Y}^{\alpha-1} \mathrm{e}^{\frac{-\mathrm{Y}}{\beta}} \mathrm{dY} \\
0<\mathrm{Y}<\infty \text { e } \alpha, \beta>0
\end{gathered}
$$

em que:

$\mathrm{G}(\mathrm{Y} \leq$ yo $)$ - probabilidade de ocorrer uma quantidade de precipitação igual ou inferior a yo

Y - variável aleatória de precipitação $(\mathrm{mm})$

$\beta \quad$ - parâmetro de escala da variável aleatória $Y$

$\alpha \quad$ - parâmetro de forma

$\Gamma(\alpha)$ - função gama

yo - valor de precipitação que tem probabilidade acumulada $\mathrm{G}(\mathrm{Y} \leq$ yo $)$ de ocorrer

Com base nos valores diários de ETo e Pp, procurou-se associar o período em que a $\mathrm{Pp}>$ ETo com o estádio de floração e frutificação da cultura de feijão para as diferentes épocas de plantio (verão, safrinha e inverno) de modo a reduzir a demanda total de água ao longo do ciclo da cultura.

Empregando os valores dos coeficientes de cultura para o feijão obtidos por Steinmetz (1984) apresentados na Tabela 2, estimou-se a evapotranspiração da cultura (ETc), utilizando-se a Eq. 3.

$$
\mathrm{ETc}=\mathrm{ETo} \mathrm{Kc}
$$

em que:

Kc - coeficiente de cultura

Tabela 2. Valores de Kc e duração dos estádios de desenvolvimento da cultura do feijão ao longo do ciclo fenológico

\begin{tabular}{lcccccc}
\hline Cultura & Estádios & I & II & III & IV & Total \\
\hline \multirow{2}{*}{ Feijão } & Duração (dias) & 15 & 20 & 40 & 15 & 90 \\
& Kc & 0,3 & 0,8 & 1,1 & 0,7 & \\
\hline Fonte: Steinmetz (1984) & & & & & &
\end{tabular}

A lâmina suplementar de irrigação foi obtida pelo balanço hídrico, considerando-se a precipitação provável e a evapotranspiração. As lâminas suplementares de irrigação e datas de plantio definidas em dia Juliano, foram espacializadas utilizando-se o aplicativo Surfer 5.0 e a krigagem com interpolador, o que permitiu a geração dos mapas temáticos.

\section{RESULTADOS E DISCUSSÃO}

A Figura 1 apresenta os valores de ETo e Pp diários estimados pelo software DEMANDA para Goiânia, na qual se pode observar o comportamento sazonal do período chuvoso. O mesmo comportamento foi verificado para as demais localidades empregadas neste estudo, típico da região do cerrado. Pela análise do comportamento sazonal da Pp, definiuse a data de plantio para que houvesse a coincidência do estádio de maior demanda de água da cultura de feijão com o período de maior incidência das chuvas, na tentativa de reduzir a lâmina suplementar de irrigação.

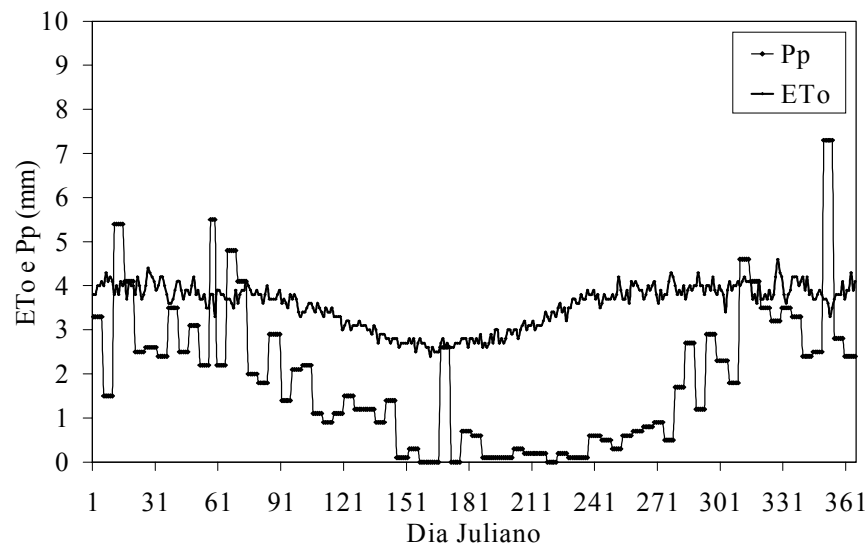

Figura 1. Comportamento sazonal da ETo e Pp para Goiânia

A Tabela 3 apresenta os valores percentuais de redução da demanda total de água para a cultura do feijão, com a adoção da irrigação suplementar, para diferentes épocas de plantio no 
Tabela 3. Demanda de água para a cultura do feijão com irrigação total e suplementar para alguns municípios do Estado de Goiás

\begin{tabular}{|c|c|c|c|c|c|c|c|c|c|}
\hline \multirow[b]{2}{*}{ Município } & \multicolumn{3}{|c|}{ Feijão Verão } & \multicolumn{3}{|c|}{ Feijão Safrinha } & \multicolumn{3}{|c|}{ Feijão Inverno } \\
\hline & $\begin{array}{c}\mathrm{IT}^{*} \\
(\mathrm{~mm})\end{array}$ & $\begin{array}{l}\mathrm{IS}^{* *} \\
(\mathrm{~mm})\end{array}$ & $\begin{array}{c}\mathrm{PR}^{* * *} \\
(\%)\end{array}$ & $\begin{array}{c}\mathrm{IT}^{*} \\
(\mathrm{~mm})\end{array}$ & $\begin{array}{l}\mathrm{IS}^{* *} \\
(\mathrm{~mm})\end{array}$ & $\begin{array}{c}\mathrm{PR}^{* * *} \\
(\%)\end{array}$ & $\begin{array}{c}\mathrm{IT}^{*} \\
(\mathrm{~mm})\end{array}$ & $\begin{array}{l}\mathrm{IS}^{* *} \\
(\mathrm{~mm})\end{array}$ & $\begin{array}{c}\mathrm{PR}^{* * *} \\
(\%)\end{array}$ \\
\hline Aragarças & 275,4 & 38,3 & 620,1 & 284,3 & 97,2 & 192,4 & 221,7 & 179,0 & 23,9 \\
\hline Catalão & 292,6 & 84,3 & 246,9 & 368,3 & 167,8 & 119,5 & 238,1 & 176,4 & 35,0 \\
\hline Formosa & 270,9 & 58,7 & 361,7 & 285,8 & 82,5 & 246,5 & 267,4 & 90,3 & 196,1 \\
\hline Goiânia & 296,9 & 59,0 & 403,1 & 304,5 & 68,4 & 345,1 & 213,8 & 151,9 & 40,8 \\
\hline Goiás & 288,0 & 33,9 & 750,3 & 291,9 & 34,0 & 757,5 & 263,1 & 116,1 & 126,5 \\
\hline Mineiros & 293,7 & 60,2 & 387,6 & 297,7 & 129,6 & 129,7 & 251,6 & 174,2 & 44,5 \\
\hline Pirenópolis & 280,8 & 36,2 & 675,0 & 345,6 & 131,5 & 162,9 & 215,9 & 134,8 & 60,2 \\
\hline Posse & 278,6 & 82,1 & 239,5 & 293,4 & 207,9 & 41,1 & 353,6 & 284,6 & 24,2 \\
\hline Rio Verde & 282,7 & 65,6 & 330,9 & 293,2 & 129,3 & 126,7 & 243,0 & 170,2 & 42,8 \\
\hline Média & 284,4 & 57,6 & 446,1 & 307,2 & 116,5 & 235,7 & 252,0 & 164,2 & 66,0 \\
\hline
\end{tabular}

* Lâmina de irrigação total

** Lâmina de irrigação suplementar

*** Porcentagem de redução da lâmina de irrigação quando se adota a irrigação suplementar

Estado de Goiás. Na referida tabela observa-se uma redução média no porcentual na demanda total de água ao longo do ciclo da cultura de feijão, de 446,$1 ; 235,7$ e $66 \%$ para o plantio de verão, safrinha e inverno, respectivamente. O porcentual de redução foi maior nos plantios de verão e safrinha, por coincidir com os períodos de maior ocorrência das chuvas, em que a irrigação é feita em curtos períodos de estiagem e no intervalo entre irrigações, em que a soma da ETc supera a Pp; o mesmo não se verifica no plantio de inverno, o que acarretou em menores valores do percentual de redução da demanda total de água na cultura do feijão.

A Figura 2 apresenta a demanda de irrigação para a safra de inverno para a região de Goiânia, considerando-se irrigação total para suprir a demanda evapotranspirométrica e a irrigação suplementar, onde se pode observar a redução na demanda de irrigação quando se adota a suplementação do regime pluviométrico local.

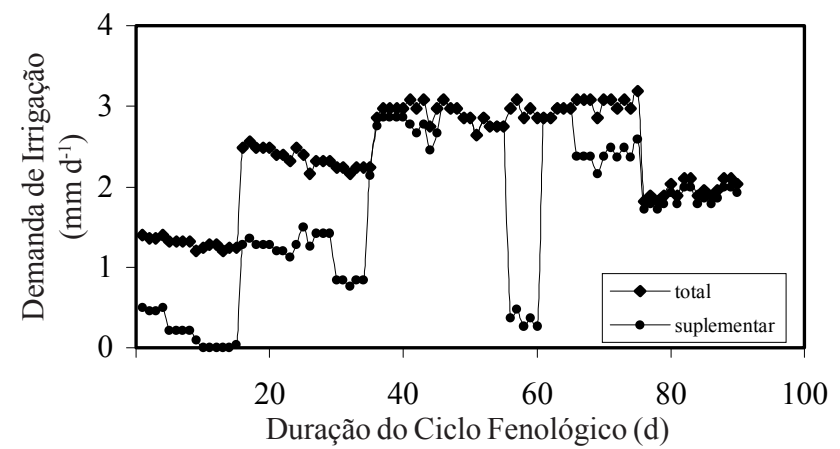

Figura 2. Demanda de irrigação do feijão para a safra de inverno, considerando-se irrigação total e suplementar para Goiânia, $\mathrm{GO}$

As Figuras 3 e 4 apresentam os mapas temáticos das lâminas suplementares máximas de irrigação, ao longo do ciclo fenológico, e data de plantio em dias julianos, para a cultura do feijão com plantios de verão, safrinha e inverno. Os mapas gerados neste trabalho servirão de orientação para os projetistas no dimensionamento de sistemas de irrigação, permitindo a elaboração de sistemas de menor porte, redução no consumo de energia e na quantidade de água necessária à irrigação.
A.

B.

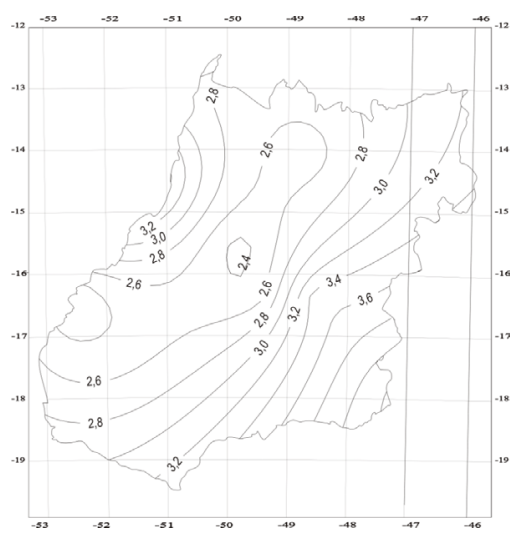

C.
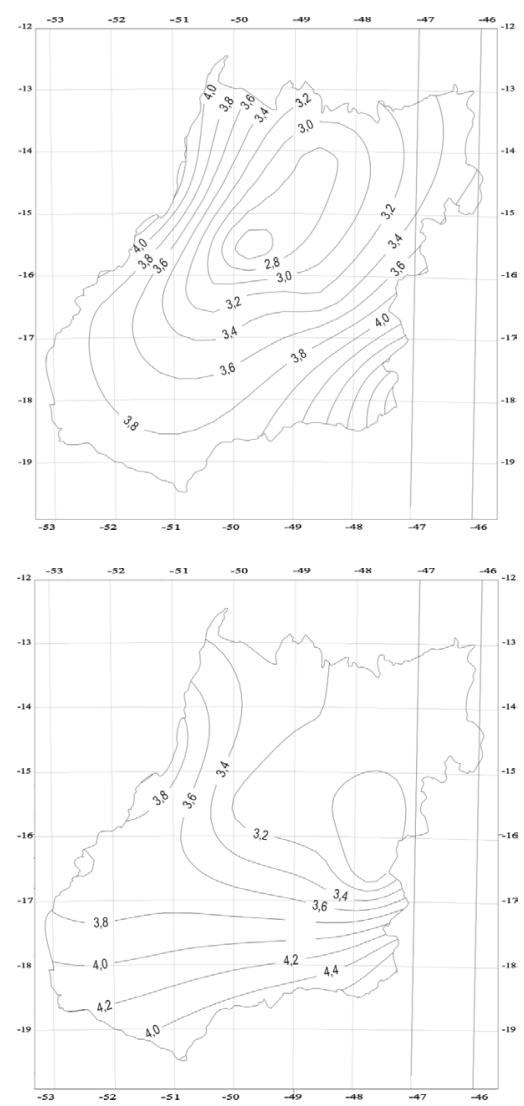

Figura 3. Lâmina suplementar de irrigação para a cultura de feijão, com plantio de verão (A), safrinha (B) e inverno (C) 
A.

B. C.
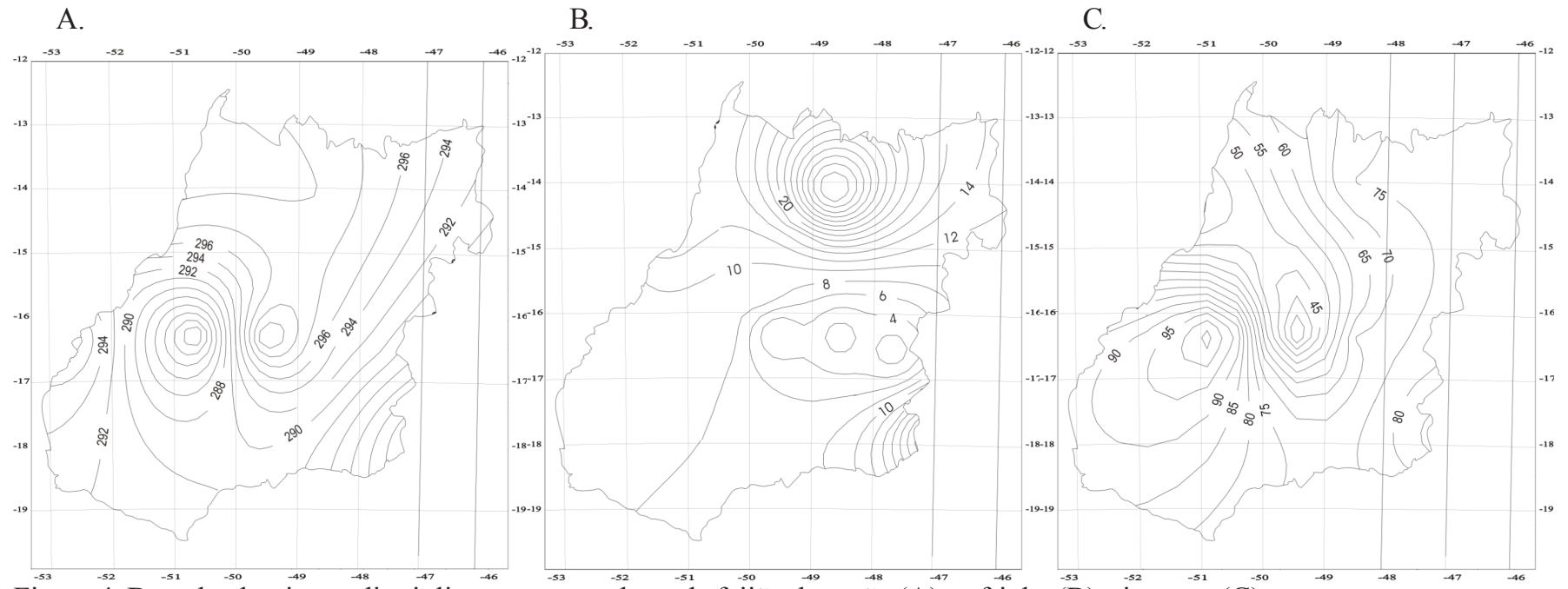

Figura 4. Data de plantio em dias julianos para a cultura de feijão de verão $(A)$, safrinha ${ }^{-53}$ (B) e inverno $(C)^{-58}$

\section{CONCLUSÃO}

A adoção da irrigação suplementar para a cultura do feijão no Estado de Goiás, com valores estimados da lâmina de irrigação empregando-se dados de uma série histórica com 15 anos de observação, permite uma redução média na quantidade total de água a ser aplicada na irrigação, nos plantios de verão, safrinha e inverno, de 446,1;235,7 e 66\%, respectivamente, e a elaboração de sistemas de menor porte, redução no consumo de energia e na quantidade de água necessária à irrigação.

\section{LITERATURA CITADA}

Assad, E.D.; Sano, E.E.; Masutomo, R. Uso de modelos numéricos de terreno na espacialização de dados pluviométricos. In: Assad, E.D.; Sano, E.E. Sistema de informações geográficas: Aplicações na agricultura. Brasília: EMBRAPASPI, 1998, p.251-309.

Assis, F.N. Ajuste da função gama aos totais semanais de chuva de Pelotas-RS. Revista Brasileira de Agrometeorologia, Santa Maria, v.1, n.1, p.131-136, 1993.

Assis, F.N.; Arruda, H.V.; Pereira, A.R. Aplicações de estatística à climatologia: teoria e prática. Pelotas: UFPel, 1996. 161p.

Bernardo, S. Manual de irrigação. Viçosa: Imprensa Universitária, 1995. 657p.
Bernardo, S.; Hill, R.W. Um modelo para determinação de irrigação suplementar. In: Ferri, M. G. Simpósio sobre o cerrado. São Paulo: EDUSP, 1979, p.255-269.

Carvalho, D.F. Otimização do uso da água no perímetro irrigado do Gorutuba. Viçosa: UFV, 1998, 145p. Tese Doutorado

Costa, M.H. Modelo de otimização dos recursos hídricos para irrigação, conforme a época de plantio. Viçosa: UFV, 1991. 111p. Dissertação Mestrado

Nascimento, J.L. Respostas de duas cultivares de feijoeiro (Phaseolus vulgaris L.) a cinco lâminas de irrigação aplicadas durante o estádio de desenvolvimento vegetativo nos sistemas de plantio direto e convencional. Goiânia: UFG, 1998, 138p. Tese Doutorado

Sediyama, G.C. Evapotranspiração: necessidade de água para as plantas cultivadas. Curso de Engenharia e Manejo da Irrigação. Brasília: ABEAS, 1996. 176p.

Sediyama, G.C.; Melo, J.S.P.; Alves, A.R. Determinação dos parâmetros da distribuição gama, em função das alturas médias mensais de precipitação dos dias chuvosos. Revista Ceres, Viçosa, v.43, n.247, p.254-266, 1995.

Silva, E.L. Relação água-solo-planta. Lavras: ESAL, 1982.30p.

Sousa, E.F. Modelo computacional aplicado ao manejo e planejamento de irrigação. Viçosa: UFV, 1993. 65p. Dissertação Mestrado

Steinmetz, S. Evapotranspiração máxima no cultivo do feijão de inverno. Goiânia: EMBRAPA-CNPAF, 1984, 4p. 